\title{
La cuina compromesa: estudio de caso exploratorio sobre la viabilidad de prácticas alternativas de restauración en la nueva ruralidad catalana
}

\section{Ignacio López-Moreno}

Universidad Autónoma Metropolitana. Unidad Lerma (UAM-Lerma).

Departamento de Procesos Sociales

i.lopez@correo.ler.uam.mx

\author{
Neus Monllor i Rico \\ Arrels a Taula \\ neus@arrelsataula.cat
}

Jaume Guillamon

Associació Aladula

aberaki@gmail.com

\section{Carles Guirado González}

Universitat Autònoma de Barcelona. Departament de Geografia carlesguirado@gmail.com

\section{F. Xavier Medina}

Universitat Oberta de Catalunya

fxmedina@gmail.com

\section{Resumen}

El nuevo paradigma agrosocial y la nueva ruralidad europea son objeto de múltiples investigaciones y discusiones académicas. El presente trabajo contribuye a ellas a través de un estudio de caso de plausibilidad que tuvo dos objetivos: a) construir una realidad empírica que nos permitiera comprobar la plausibilidad de lo descrito en las teorías de la nueva ruralidad, y $b$ ) definir los puntos fuertes y débiles de la misma a través de la socialización de la experiencia con los actores principales de las redes construidas en la primera fase. Mediante una metodología cualitativa que duró tres años se demostró la viabilidad de un espacio gastronómico alternativo que siguiera los principios de la cuina compromesa (cocina comprometida), y mediante un proceso participativo se definieron sus puntos fuertes y débiles. El artículo concluye subrayando la capacidad de transformación territorial de las nuevas prácticas gastronómicas que están surgiendo en Cataluña.

Palabras clave: cuina compromesa; economía rural; nueva ruralidad 
Resum. La cuina compromesa: estudi de cas exploratori sobre la viabilitat de pràctiques alternatives de restauració a la nova ruralitat catalana

El nou paradigma agrosocial i la nova ruralitat europea són objecte de múltiples investigacions i discussions acadèmiques. Aquest treball hi contribueix mitjançant un estudi de cas de plausibilitat que va tenir dos objectius: $a$ ) construir una realitat empírica que ens permetés comprovar la plausibilitat del que s'ha descrit a les teories de la nova ruralitat, i b) definir els seus punts forts i febles mitjançant la socialització de l'experiència amb els actors principals de les xarxes construïdes a la primera fase. Mitjançant una metodologia qualitativa que va durar tres anys, es va demostrar la viabilitat d'un espai gastronòmic alternatiu que seguís els principis de la cuina compromesa, i mitjançant un procés participatiu se'n van definir els punts forts i febles. L'article es tanca subratllant la capacitat de transformació territorial de les noves pràctiques gastronòmiques que sorgeixen a Catalunya.

Paraules clau: cuina compromesa; economia rural; nova ruralitat

Résumé. La cuina compromesa: Étude de cas exploratoire sur la faisabilité des pratiques de restauration alternatives dans la nouvelle ruralité catalane

Le nouveau paradigme agrosocial et la nouvelle ruralité européenne font l'objet de multiples enquêtes et discussions académiques. Ce travail contribue à étudier ces aspects à partir d'une étude de cas de plausibilité qui a pour but deux objectifs: (a) la construction d'une réalité empirique qui nous permettrait de vérifier la plausibilité de ce qui est décrit dans les théories de la nouvelle ruralité, et (b) définir les forces et les faiblesses de cette réalité par le biais de la socialisation de l'expérience avec des acteurs majeurs dans les réseaux construits dans les premiers temps de la recherche. Grâce à une méthodologie qualitative menée à bien durant trois ans, la faisabilité d'un espace gastronomique alternatif pour suivre les principes de ce que l'on a appelé la cuina compromesa (cuisine engagée) démontre ses forces et ses faiblesses, à travers un processus participatif et défini. L'article conclut en insistant sur la capacité de transformation territoriale des nouvelles pratiques culinaires qui émergent en Catalogne.

Mots-clés: cuina compromesa; économie rurale; nouvelle ruralité

Abstract. Cuina Compromesa: An exploratory case study on the viability of alternative for restaurant practices in Catalonia new rurality

The new agrosocial paradigm and the new European rurality are subject to much research and academic discussion. This paper aim to make a contribution with a case study on their empirical viability by: (a) designing and implementing an empirical situation based on theoretical assumptions, and (b) defining the strengths and weaknesses of this initiative by matching the findings with the key actors involved in the project. The research used a qualitative methodology for three years, and demonstrated that there is space for an alternative restaurant model that follows the principles of cuina compromesa (committed cooking). The article concludes underlining the transformative capacity of the new gastronomic practices in Catalonia.

Keywords: cuina compromesa; rural economy; new rurality 


\section{Sumario}
1. Introducción
4. Resultados
2. Marco teórico
5. Conclusiones
3. Metodología
Referencias bibliográficas

\section{Introducción}

Europa en general y Cataluña en particular están inmersas en un proceso de cambio de referente en las prácticas agroalimentarias desde finales del siglo pasado. Las últimas décadas han supuesto un cuestionamiento de las prácticas de producción, distribución y consumo alimentario que habían sido abandonadas en manos de la gran industria y las corporaciones multinacionales. Numerosos estudios han analizado el cambio en sí mismo (Burch y Lawrence, 2009; Civitello, 2008; Contreras y Ribas, 2012; Fonte, 2013; Marsden, 2000; Medina, 2010; Nützenadel y Trentmann, 2008; Pechlaner y Otero, 2008; Pollan, 2014; Standage, 2009), el por qué (Beck, 1998; Guidonet, 2010; Levenstein, 2012; Menasche, 2003; Ogle, 2009; Paddock, 2015; Ploeg, 2010; Vázquez y Medina, 2015) y el cómo (Ayora, 2012; Díaz, 2005; Holt y Shattuck, 2011; Morales, 2011, Nestle, 2007; Sgarbi y Menasche, 2015).

La revisión de estos trabajos permite vislumbrar la aparición de un nuevo paradigma agrosocial basado en la escala local, la diversidad, el medio ambiente, la cooperación, la innovación, la autonomía, el compromiso social y la ralentización productiva ${ }^{1}$ (Monllor, 2011). Tal y como Ploeg y Renting (2004) describen, este cambio de paradigma no tiene lugar de la noche a la mañana ni de manera consensuada, sino a través de experimentos y nuevas prácticas que van creando un nuevo sentido y una ética de la acción social.

Este trabajo busca contribuir a las teorías que defienden la existencia de ese nuevo paradigma desde una aproximación exploratoria con un estudio de caso de plausibilidad. Para ello se partió de la siguiente hipótesis: si las contribuciones teóricas y empíricas sobre la existencia de un nuevo paradigma agrosocial son ciertas, debe existir un espacio para un nuevo modelo de hostelería que esté en consonancia con el mismo. El nuevo modelo de hostelería fue construido heurísticamente y definido como cuina compromesa ${ }^{2}$ (Monllor et al., 2014). Este modelo tiene cinco principios básicos que rigen su funcionamiento: $a$ ) compra local, $b$ ) producción ecológica, $c$ ) alimentos de temporada, $d$ ) contacto directo y $e$ ) pagos justos.

Por lo tanto, la investigación tuvo dos objetivos principales: a) construir una realidad empírica que nos permitiera comprobar la plausibilidad de lo

1. En el siguiente apartado se realizará una descripción más profunda del mismo.

2. Aunque este trabajo ha sido escrito en español, el equipo ha decidido no traducir la expresión del catalán para preservar las connotaciones de origen y el contexto sociocultural que vieron nacer y perfilar esta práctica. 
descrito en las teorías de la nueva ruralidad, y $b$ ) definir los puntos fuertes y débiles de la misma a través de la socialización de la experiencia con los actores principales de las redes construidas en la primera fase. Esto conllevó el diseño y la implementación de una investigación basada en el método de investigaciónacción participativa, durante la cual se implementaron fundamentalmente técnicas cualitativas de recolección de datos basadas en muestreos no probabilísticos (como el muestreo en cadena o bola de nieve), principalmente observación participante y grupos de discusión. Como estrategia de investigación se usó el estudio de caso, basado en la creación del Espai Tomata como unidad de observación en Burg, un pueblo del Pirineo de Lérida.

\section{Marco teórico}

\subsection{El nuevo paradigma agrosocial}

La Europa rural del siglo xxi es, entre otras cosas, el resultado tanto de los procesos de intensificación de la producción primaria como de los de abandono de la actividad agraria que tuvieron lugar en la segunda mitad del siglo xx (Cloke, 2006; Woods, 2005). La Revolución Verde impactó de manera directa en el espacio rural y en el sistema de producción agroalimentaria tradicional, lo que provocó una profunda reestructuración de las economías agrarias y rurales, resultado que se muestra en numerosos paisajes europeos (Arqué et al., 1982; Bowler, 1996; Lockwood, 1999).

Las pequeñas explotaciones familiares que conformaban la base de la economía agraria de subsistencia fueron desapareciendo progresivamente por la dificultad de ser mecanizadas y por sus bajos índices de productividad en relación con aquellas tierras que ya incorporaban el uso de maquinaria agrícola y tratamientos químicos para controlar y hacer más eficientes los cultivos (Ploeg, 2006). Los cambios en los sistemas agrarios y en la organización del trabajo agrícola provocaron en muchos territorios rurales una profunda crisis estructural, frente a la cual muchos de sus habitantes optaron por migrar hacia las áreas urbanas (Camarero, 1993). La despoblación del campo tuvo dramáticos efectos sobre la organización social, la cultura local y el territorio, impactos que hoy en día aún se pueden observar como una huella en el paisaje y en la sociedad rural (Aldomà et al., 2004).

La respuesta al desequilibrio producido por los procesos de intensificación productiva en el campo provoca que a partir de los años setenta se empiece a articular en algunas zonas europeas el concepto de desarrollo rural (Viladomiu et al., 2009). Este sería integrado, endógeno o sostenible, en función de la visión global de la idea de desarrollo, pero todos ellos con el objetivo común de revitalizar las zonas rurales acechadas por el abandono y la marginalidad.

Las prácticas de desarrollo rural evolucionan y se reinventan, hasta que en el año 2006 la OCDE publica "The New Rural Paradigm: policies and governance», una aproximación teórica a la nueva realidad de los territorios rurales en Europa. Una mirada abierta a las oportunidades que las economías 
rurales poseen en un mundo cada vez más globalizado y, en parte, más vinculado a la localidad del territorio. La OCDE argumenta el paso de un paradigma agroindustrial a un nuevo escenario mucho más multifuncional.

Desde un marco teórico elaborado directamente en relación con esta perspectiva, Monllor (2011) identifica la nueva ruralidad como un cambio hacia un nuevo paradigma agrosocial, un modelo de desarrollo en el que los temas sociales y agrarios se articulan como estratégicos para fortalecer las raíces del desarrollo rural. Más allá de lo estrictamente rural, el paradigma agrosocial se articula a partir de ocho elementos que caracterizan este nuevo marco teórico en el que tienen lugar nuevas prácticas. Metodológicamente, Monllor parte de la idea de la OCDE sobre el nuevo paradigma rural (OCDE, 2006), modelo que enriquece con las aportaciones de autores como Acosta (2008), Bove y Dufour (2003), Bryden (2000) y Ploeg (2008), todos ellos coincidentes en la necesidad de articular modelos teóricos nuevos que respondan a las realidades emergentes en los espacios rurales europeos. El nuevo paradigma agrosocial propuesto responde a los siguientes componentes: 1) escala local, 2) diversidad, 3) medio ambiente, 4) cooperación, 5) innovación, 6) autonomía, 7) compromiso social y 8) decrecimiento.

El paradigma de la nueva ruralidad europea responde al nuevo paradigma agrosocial que Monllor propone. Cada uno de los componentes que dan forma al nuevo paradigma emergente alude a múltiples prácticas y realidades que se están dando hoy en día en las zonas rurales europeas. Por ejemplo, el componente de la escala local hace referencia a volver a valorar el lugar como un espacio auténtico, lleno de identidad y generador de oportunidades, donde los recursos próximos se convierten en una fuente única de desarrollo endógeno y se reconoce que existe un conocimiento y una sabiduría autóctona. Dar importancia a la escala local es una manera directa de poner en marcha medidas de desarrollo rural desde el mismo territorio, ya que practicar una agricultura y una ganadería conscientes de la localidad aporta un valor añadido de primer orden.

En el desarrollo de un modelo agrosocial nacen dos conceptos innovadores que se manifiestan en los territorios rurales (aunque también en los urbanos): el nuevo campesinado y la cocina comprometida.

\subsection{El nuevo campesinado emergente}

El concepto de nuevo campesinado (new peasantry) fue establecido por Ploeg (2008) y ha sido estudiado y analizado por otros investigadores desde diferentes perspectivas (Aurélie, 2007; Binimelis et al., 2008; Euskadiko Gazteriaren Kontseilua, 2010; Gillespie y Johnson, 2010; Mailfert, 2007; Monllor, 2011; Niewolny y Lillard, 2010; Pérez, 2010). El argumento principal del fenómeno emergente muestra cómo el campesinado, lejos de desaparecer o extinguirse, se renueva y renace en infinitos lugares del mundo. El proceso de industrialización que ha desterritorializado buena parte de la agricultura es el que al mismo tiempo ha creado espacios de resistencia contra los «imperios alimentarios» 
(Ploeg, 2008: 123) que amenazan con sus estrategias agresivas a los sistemas agroalimentarios locales.

En la presente investigación hemos utilizado el concepto de nuevo campesinado para referirnos a las múltiples y diversas manifestaciones productivas que se están articulando en el territorio. Desde una óptica de desarrollo rural y de equilibrio territorial, las experiencias que muestra el nuevo campesinado están totalmente vinculadas a los motores de la dinamización local, como son la generación de empleo, el respeto por el medio ambiente y la función social en las zonas rurales (Monllor et al., 2005; O'Connor, et al., 2006; Regidor, 2008; Viladomiu, 2003).

Las experiencias agrarias del nuevo campesinado incorporan los elementos económicos, sociales y ambientales que definen el paradigma agrosocial, con lo que las nuevas empresas agrarias tienden a potenciar el dinamismo territorial desde sus propios recursos. En términos de estrategia, hay cuatro elementos que caracterizan las actividades económicas del nuevo campesinado: 1) dimensión física y económica pequeña, 2) venta en circuitos cortos, 3) diversificación de la actividad agraria y 4) producción ecológica. Según Monllor (2013: 12):

Los nuevos campesinos están arraigados en la localidad, creen en la diversificación, promueven prácticas respetuosas con el medio ambiente, cooperan para avanzar en su causa común, introducen tecnologías apropiadas y de escala humana, luchan por su propia autonomía, tienen en cuenta a las generaciones futuras y tratan de reducir la intensificación respecto a las prácticas agrícolas convencionales.

Es importante tener en cuenta que el nuevo campesinado está formado tanto por descendientes de familias agrarias tradicionales como por personas, algunas de origen urbano, procedentes de familias no campesinas. Lo que las identifica es la proximidad a un modelo agrosocial que les permita cubrir sus necesidades y ofrecer alimentos en los que poder confiar.

\subsection{La cuina compromesa}

El concepto de cuina compromesa lo establecieron Monllor y su equipo basándose en una exhaustiva revisión de la bibliografía y en una experiencia empírica (2014). Es un concepto innovador en el que se incluye una perspectiva territorial y agraria de la alimentación y del poder del consumo en general, y en particular, en los restaurantes. Comer es una acción directa de política agraria que modifica el espacio socioeconómico en función de las decisiones, individuales o colectivas, que se tomen (Petrini, 2005; Vivas, 2014). La responsabilidad de elegir el modelo agrario de un territorio está estrechamente vinculada con el acto cotidiano de comer. La cuina compromesa reivindica esta visión desde el análisis y la observación de las dinámicas actuales en Cataluña y en la presente investigación se analizan sus vínculos con el nuevo campesinado.

La cuina compromesa se define, como habíamos señalado más arriba, a partir de cinco variables: 1) compra local, 2) producción ecológica, 3) alimentos de 
temporada, 4) contacto directo y 5) pagos justos (Monllor et al., 2014). Estos cinco puntos de partida son los que comparte de manera natural con el nuevo campesinado, hecho que permite el fortalecimiento de nuevas dinámicas de crecimiento y de reproducción social.

La desestructuración de los sistemas agroalimentarios locales forzada por el modelo agroindustrial y asumida en ocasiones como inevitable es rebatida por una infinidad de experiencias agroalimentarias donde el territorio vuelve a ser tenido en cuenta como la base imprescindible de producción y consumo (Vivas, 2010). La deslocalización productiva se difumina a favor de una reivindicación de los alimentos con garantía en su origen y en su calidad (Aguilar y Lozano, 2008; López, 2014; López y Aguilar, 2012; López y Aguilar, 2013; López y Pérez, 2014; Lozano y Aguilar, 2010; Renting et al., 2003).

La cuina compromesa debe ser entendida como una de las cristalizaciones regionales de la nueva ruralidad europea y de la reinvención de lo rural. En cierto sentido, estamos ante una nueva forma económica que tiene un cuerpo ético y moral desde el que guía su práctica, lo que podría ser una nueva forma de economía moral actualizada a la nueva ruralidad (Scott, 1976).

A modo de síntesis, podríamos decir que estos dos elementos, nuevo campesinado y cuina compromesa, dan forma a un motor de transformación territorial a favor de nuevas articulaciones que tiene como objetivo la generación de un bienestar local mediante la dignificación de la producción y la elaboración de alimentos de calidad en consonancia con los agroecosistemas locales ecológicamente adaptados.

\section{Metodología}

El presente artículo es fruto de una investigación que tuvo dos objetivos secuenciales: a) construir una realidad empírica que nos permitiera comprobar la plausibilidad de lo descrito en las teorías de la nueva ruralidad con respecto a la hostelería, y b) definir los puntos fuertes y débiles de la misma a través de la socialización de la experiencia con los actores principales de las redes construidas en la primera fase. Para ello, seguimos un planteamiento metodológico de tipo cualitativo, con una orientación naturalista y un método de estudio de caso. Elegimos la metodología cualitativa porque permite responder a preguntas de cómo y por qué ocurren los fenómenos sociales en un contexto creado (la unidad de observación), pero no controlado. La orientación naturalista viene por la segunda característica de la unidad de observación: la ausencia de control sobre el espacio y las acciones de los sujetos que en él interactúan una vez creado nos permiten observar de manera participativa y comprender los comportamientos sociales en el día a día.

La estrategia de estudio de caso fue elegida por la facilidad que otorga a estudios de tipo exploratorio. Eckstein propone cinco categorías de estudio según su capacidad de desarrollo teórico (citado por Rivera y Tonatiuh, 2009: 271): a) estudios configurativo-ideográficos, $b$ ) estudios configurativo-ordenados, $c$ ) estudios de caso heurísticos, $d$ ) pruebas de plausibilidad, y e) estudios de caso cruciales. Nuestra investigación se construyó como un estudio de caso 
del cuarto tipo: prueba de plausibilidad. Este modelo de estudio de caso busca someter a examen teorías que se han formulado previamente por medio de otros estudios de caso e investigaciones.

La investigación tuvo cuatro etapas principales: a) diseño del estudio de caso, $b$ ) selección y construcción de la unidad de observación, $c$ ) recolección de datos en la unidad de observación, y d) socialización de los datos en grupos de discusión. La primera etapa supuso la lectura de diversos manuales y estudios sobre metodología de investigación-acción participativa para obtener información sobre la historia y el funcionamiento de esta metodología (Geilfus, 2002; Green y Thorogood, 2009; Hart y Bond, 1995). Si bien es cierto que esta metodología suele tener más énfasis en la acción transformadora que en la investigación (Koch y Kralik, 2006), la combinación con el tipo de método de estudio de caso aseguró la consecución de los objetivos marcados.

La segunda etapa, directamente basada en los planteamientos básicos ya mencionados de Monllor (2011), comenzó con la elección de Burg en la comarca pirenaica del Pallars Sobirà (Lérida) como el lugar idóneo para la construcción de la unidad de observación: el Espai Tomata. Burg resultó el lugar adecuado para construir el restaurante por su ubicación, a menos de dos horas de importantes núcleos de población (Barcelona, Lérida o Zaragoza), un marco natural privilegiado (situado a las puertas del parque natural del Alto Pirineo y cerca de la estación de esquí de Port Ainé), y una población cercana a los veinte habitantes. Estas características coincidían con la construcción social de lo rural que caracteriza a la nueva ruralidad y la hace deseable como objeto de consumo simbólico (Aguilar, 2014; Anjos y Caldas, 2014; López y Thomé, 2015). El restaurante se inauguró en el año 2010 y siguió los cinco principios de la cuina compromesa en su funcionamiento.

La tercera etapa se llevó a cabo a través de la técnica de observación participante fundamentalmente (Guasch, 1996; Pujadas et al., 2010). Durante tres años el restaurante estuvo abierto y fue lugar de encuentro para productores que respondían al esquema del nuevo campesinado y consumidores que buscaban una oferta gastronómica diferente. La experiencia del día a día sobre las necesidades y potencialidades del esquema de la cuina compromesa fueron recogidas en cuadernos de campo individuales por parte de los dos principales investigadores de campo. La construcción de redes de proveedores y consumidores fue un proceso lento y de crecimiento exponencial que hizo que la unidad de observación se extendiera por casi toda Cataluña. Esta fase constató con detalle el surgimiento del nuevo campesinado en la comarca del Pallars Sobirà y en territorios circundantes, situados en el área de influencia del Espai Tomata.

La cuarta etapa consistió en la socialización de los datos obtenidos por los investigadores en unas sesiones de trabajo tituladas «Nova pagesia i cuina compromesa». Estas sesiones siguieron el esquema de los grupos de discusión conscientes, y reunieron, a través de una metodología no probabilística (Brunet, et al., 2002), a los actores principales de la red de producción y consumo de la que el Espai Tomata era núcleo. Las jornadas de trabajo tuvieron lugar los días 16 y 17 de mayo de 2013 y reunieron en Burg a un total de 48 personas 
provenientes de diferentes partes de la geografía catalana. Durante las mismas se intercambiaron experiencias subjetivas e intersubjetivas sobre las nuevas prácticas que estaban surgiendo en el territorio catalán y que respondían total o parcialmente al modelo de la cuina compromesa.

Los perfiles de las personas que participaron respondían a tres categorías: productores, restauradores y cocineros, y técnicos relacionados con la cadena de distribución de alimentos. Las jornadas formaron parte del Plan Anual de Transferencia Tecnológica (PATT) del Departamento de Agricultura, Ganadería, Pesca y Alimentación de la Generalitat de Catalunya, lo que nos permitió reunir a personas implicadas y especializadas, caracterizadas en su mayoría por tener un buen conocimiento de las nuevas prácticas sociales en torno a la agricultura, la alimentación y la restauración que estaban surgiendo en Cataluña.

$\mathrm{Al}$ inicio de las jornadas se realizaron una serie de ponencias a cargo de expertos sobre temas relacionados con la cuina compromesa, como son el movimiento agroecológico y de soberanía alimentaria, la huella ecológica de los alimentos o el uso de transgénicos en la agricultura y cómo estas acciones afectan a la salud de las personas. El objetivo de estas ponencias fue dotar de elementos de debate a las personas asistentes para las posteriores sesiones participativas de diagnosis y de propuestas estratégicas. A continuación, se realizaron sesiones participativas y grupos de discusión, guiados por dos especialistas en técnicas de participación, en los que se elaboró un análisis DAFO del nuevo campesinado y la cuina compromesa, que posteriormente se plasmaría en un flujograma, a partir del cual se identificaron, priorizaron y relacionaron los principales problemas y limitaciones con los que se encontraba el sector. Finalmente, una vez detectados los problemas clave, se trabajó en el diseño de algunas líneas estratégicas para fortalecer la cuina compromesa. La finalidad de esta última sesión participativa era explorar posibles acciones para solucionar los problemas que se identificaron como prioritarios, así como disponer de un primer marco de actuación a partir del cual empezar a trabajar conjuntamente y establecer futuras líneas de trabajo.

Las jornadas fueron un punto de encuentro entre productores y restauradores, lo que sirvió para potenciar la creación de sinergias entre actores de ambos sectores que buscan modelos gastronómicos alternativos. Además, dotaron a la investigación de un valioso material para el análisis, del cual se derivaba un importante volumen de información útil para aproximarnos, de modo exploratorio, a la implementación de la cuina compromesa en Cataluña, a la tipificación de los actores que intervienen en la misma y a los relatos que se desprenden de esta nueva identidad colectiva. Los resultados del tratamiento, análisis e interpretación de la información generada a partir de este encuentro se exponen con detalle en el siguiente apartado.

\section{Resultados}

En este apartado se recogen los resultados obtenidos durante la investigación. La producción de estos datos es fruto de un diálogo entre los diferentes actores 
que participaron en las jornadas Nova Pagesia i Cuina Compromesa, entre los que se incluye el equipo de investigación. Por lo tanto, estos resultados son fruto de un proceso dialógico entre actores privilegiados que se materializan en una narrativa intersubjetiva. Esta narrativa se ofrece de la manera más pura posible, sin jerarquías internas ni interpretaciones posteriores, para preservar la naturaleza de la metodología que caracteriza a la investigación-acción participativa (Geilfus, 2002; Green y Thorogood, 2009).

La identificación de los puntos fuertes y débiles de la cuina compromesa como modelo alternativo para la gastronomía en Cataluña puso de manifiesto los engranajes sobre los que se puede avanzar para reorganizar las prácticas agroalimentarias en consonancia con el nuevo paradigma agrosocial que forma parte de la nueva ruralidad. Fomentar los puntos fuertes y corregir los débiles puede formar parte de una estrategia territorial que sirva para ampliar los espacios y nichos existentes, así como para redirigir y reconvertir los que se encuentran dentro del viejo paradigma productivista.

\subsection{Puntos fuertes de la cuina compromesa}

Los puntos fuertes de la cuina compromesa definidos durante las jornadas celebradas en Burg se pueden agrupar en cuatro categorías principales: $a$ ) el valor de lo local, $b$ ) la confianza en la calidad, $c$ ) la cultura gastronómica catalana, y d) la capacidad organizativa de los actores.

Uno de los valores fundamentales sobre los que se apoya la cuina compromesa es la articulación de sus estrategias a partir de la escala local. Las sesiones de trabajo identificaron el valor fundamental que incorpora la proximidad geográfica y facilitaron la relación entre la producción y el consumo, ya sea en términos sociales, territoriales o culturales. Esta reflexión conllevó argumentar que esto implica una reducción de costes para mover los alimentos, lo cual se transforma en una externalidad positiva tanto en términos económicos como ecológicos. Dentro de la escala local también se detalló la valorización del producto de proximidad identificado con quien lo produce, y ello como un valor agregado simbólico al producto. Por otro lado, la escala local de la cuina compromesa implica un aumento en la demanda de producto local que ayuda a la consolidación de las empresas rurales y a la territorialización de buena parte de la cadena.

En segundo lugar, se identificaron una serie de elementos en relación con la confianza en los alimentos y su calidad. Los principios agroecológicos requeridos por la cuina compromesa mejoran la seguridad y la calidad de los ingredientes empleados en la cocina. En este sentido, el respeto a las estaciones y los procesos de maduración naturales puede suponer una mejora en la calidad nutricional y organoléptica de los alimentos. Esto puede suponer el redescubrimiento de sabores con identidad propia, así como de placeres organolépticos que se creían olvidados o accesibles solo para personas especialistas o de alto poder adquisitivo. Se facilita, de esta forma, la socialización de ciertos valores gastronómicos ante los que, por otra parte, la producción industrial no puede ofrecer mejoras. 
La cultura gastronómica catalana arraigada en el territorio se identificó como otro de los elementos fundamentales que permite valorar la calidad y el origen de los alimentos en la cuina compromesa. Es un valor presente en toda Cataluña, que goza de una cultura gastronómica muy vinculada a su territorio, que ha emprendido diferentes iniciativas de patrimonialización gastronómica (VV. AA., 2011) y cuya cocina tradicional prepara actualmente su candidatura como patrimonio cultural inmaterial ante la UNESCO (Medina y Solanilla, 2013).

Finalmente, las sesiones de trabajo identificaron el crecimiento de empresas de marcado carácter social como el vehículo ideal para organizar la producción y distribución de los alimentos hacia los mercados. Se identificaron también otras redes alternativas exitosas con principios similares a la cuina compromesa, como la red de restaurantes $\mathrm{Km} 0$ de slow food.

Los puntos fuertes identificados esbozan qué elementos de la nueva ruralidad cabe potenciar y reforzar cuando hablamos del futuro de la cuina compromesa como modelo alternativo a extender. Las estrategias locales basadas en la calidad, así como los proyectos de base gastronómica y con un marcado carácter social, son los que están dibujando los nuevos escenarios rurales europeos. La articulación de sus agentes, así como las redes que crean y dinamizan, forman parte también de la nueva realidad social comprometida.

\subsection{Puntos débiles de la cuina compromesa}

Los puntos débiles del modelo de la cuina compromesa se pueden agrupar en tres categorías diferentes: $a$ ) dificultades comerciales, $b$ ) producto de consumo ocasional, $c$ ) invisiblidad institucional.

El primero de los puntos débiles de la cuina compromesa es el reto de la comercialización. En primer lugar, hay que tener en cuenta que el diálogo entre las personas que producen y las que consumen no siempre es tan fluido como se quisiera. La interacción entre los actores de la cadena suele estar mediada debido a la complejidad de las tareas de cada eslabón, con lo que la información sobre calidades y necesidades no suele fluir como sería necesario. Este hecho dificulta la organización comercial y frena la posibilidad de que los productos agrarios de calidad lleguen directamente a los restaurantes, que podrían ponerlos en valor. Por último, se destacó la dificultad de los pequeños productores de asegurar el volumen y la calidad de su producción en los tiempos y formas que se les solicitan.

La segunda debilidad es compartida con otras redes alternativas agroalimentarias, como la de los productos con denominaciones de origen protegida u orgánicos, y es que su consumo es ocasional y esporádico, no forma parte del día a día. La sociedad catalana y las sociedades occidentales en general se mueven sobre la base de unos patrones alimentarios estructurados en dos tiempos: la cotidianidad del día laboral y la especificidad del fin de semana y las fiestas. La cuina compromesa es percibida por los consumidores como una oferta del segundo segmento, aunque su vocación es entrar también en 
el primero. La vinculación al territorio agrario, aunque no siempre, tiende a ubicar a los restaurantes en espacios rurales y no urbanos, con lo que el acercamiento de gran parte de los potenciales consumidores se produce en fines de semana y fiestas.

Finalmente, se identificó una invisibilidad institucional de este tipo de iniciativas, así como la ausencia de un nicho protegido para dotarlo de seguridad durante su fase de experimentación e institucionalización. Si bien es cierto que el apoyo de las instituciones catalanas, españolas y europeas a prácticas surgidas dentro del esquema de la nueva ruralidad ha sido importante, parece que existe un agotamiento del modelo y un replanteamiento de la responsabilidad gubernamental en favor de modelos de corte neoliberal.

Los puntos débiles muestran las principales necesidades identificadas por los agentes implicados. La comercialización se identifica como la piedra angular que impide avanzar en la consolidación y expansión del modelo de la cuina compromesa. Esta está acompañada por la percepción de la cuina compromesa por parte del consumidor como un espacio o producto de consumo esporádico que no puede entrar en su cotidianeidad alimentaria. Por último, es necesario subrayar la falta de visión holística del problema del campo y lo rural por parte de las instituciones gubernamentales, así como programas y planes de desarrollo rural, que en la mayoría de los casos no consideran como elemento importante del desarrollo rural y territorial en la hostelería.

\section{Conclusiones}

El objetivo de este trabajo se planteaba desde el inicio como una contribución a las teorías que defienden la existencia de un nuevo paradigma alimentario, tanto productivo como gastronómico, desde una aproximación exploratoria a partir de un estudio de caso de plausibilidad: el Espai Tomata de la localidad de Burg, en el Pirineo de Lérida.

A través de este estudio de caso, hemos podido identificar determinados puntos fuertes y débiles de la cuina compromesa en tanto que modelo alternativo para la gastronomía en Cataluña. Entre los puntos fuertes, podemos destacar los siguientes:

a) El valor de lo local: la proximidad geográfica facilita la relación entre la producción y el consumo en términos sociales, territoriales y culturales. Implica una reducción de costes y permite una identificación directa del alimento con el productor, lo que añade valor al propio producto y contribuye a territorializar la cadena alimentaria.

b) La confianza en la calidad: lo expuesto en el apartado anterior redunda en una mayor confianza en la calidad del producto y de su resultado gastronómico, basada en el respeto de las temporalidades (estacionalidad, maduración natural...), un mejor conocimiento de la cadena alimentaria desde la producción hasta el plato, así como una puesta en valor que es directamente proporcional a una mayor implicación de los territorios locales en todo el proceso. 
c) La cultura gastronómica catalana: todo este entramado aúna lo local con una pertenencia cultural identificable y explícita que permite abundar en el estudio de los distintos productos y platos, y establecer vinculaciones patrimoniales que suponen, en sí mismas, un nuevo valor añadido a distintas partes de la cadena.

d) La capacidad organizativa de los actores: el entramado expuesto permite la creación de empresas locales de carácter social que organizan la producción y distribución de los alimentos hacia los mercados, así como otras redes alternativas con principios similares que, en conjunto, trabajan mediante una articulación efectiva de los distintos agentes.

La investigación ha puesto de manifiesto que existe en Cataluña una realidad emergente donde las personas que producen y las que consumen alimentos vinculados al territorio están cada vez más organizadas y son más conscientes de la red que están creando, y ello tiene una incidencia directa en relación con este tipo de consumo. Sin embargo, se identifican también claramente determinados puntos débiles ligados a esta iniciativa, y que nos llevan a las dificultades comerciales, empezando por la distribución y venta del producto fuera de los circuitos más habituales o establecidos; la difícil circulación de la información y su dificultosa recepción por parte del consumidor; la necesidad de acercarse al territorio (rural, principalmente) para consumir este tipo de productos; $y$, en consecuencia, el consumo ocasional de este tipo de productos, ligado más a ocasiones especiales que no al día a día. Finalmente, se observa una cierta invisibilidad institucional y de protección en relación con este tipo de iniciativas, cosa que no favorece en absoluto su implantación.

A pesar de los últimos aspectos destacados, la situación parece llevarnos actualmente hacia un nuevo escenario que permite que afloren actores que en el pasado eran tratados de alternativos, que se situaban al margen de los sistemas clásicos de producción y distribución de los alimentos, y que hoy están encontrando vías para vencer las dificultades iniciales. En este mismo sentido, algunas propuestas gastronómicas incipientes (como el propio Espai Tomata de Burg) empiezan a abrir una brecha de dinamización económica en la medida en que tejen alianzas con las experiencias agrarias cercanas y llevan a cabo una nueva propuesta integral en torno a la alimentación.

En este mismo sentido, el nuevo campesinado y la cuina compromesa dan forma a un motor de transformación territorial a favor de nuevas articulaciones, y que tiene como objetivo la generación de un desarrollo territorial integrado y de bienestar local mediante la dignificación de la producción y la elaboración de alimentos de calidad en consonancia con los agroecosistemas locales ecológicamente adaptados. Se trata, pues, de una forma de economía ética actualizada en relación con la nueva ruralidad cuya vocación es claramente social y comprometida, y cuyos frutos, si bien se presentan a través de sus destacables resultados gastronómicos, van mucho más allá de la alimentación en sí misma. 


\section{Referencias bibliográficas}

Acosta, I. L. (2008). «The paradigm of new rurality as the axis of public policies. What can we expect?». Revista Electrónica Zacatecana sobre Población y Sociedad, (8) 32, 1-20.

Aguilar, E. (2014). «Los nuevos escenarios rurales. De la agricultura a la multifuncionalidad». Éndoxa: Series Filosóficas, 33, 73-98.

Aguilar, E. y Lozano, C. (2008). «El territorio y las producciones de calidad como factor de desarrollo sostenible». En: Fundación de Estudios Rurales. Agricultura Familiar en España 2008: Territorio, agricultura y medio ambiente. Córdoba: UPA, 170-173.

Aldomá, I.; Mendizábal, E.; Pèlachs, A. y Soriano, J. M. (2004). «La transformació del territori i del paisatge de l'Alt Pirineu». En: Vicendo, E. (coord.). Medi, territori i història. Les transformacions territorials en el món rural català occidental. Lérida: Pagès.

Anjos, F. S. DOS y Caldas, N. V. (2014) «Da medida do rural ao rural sob medida: representações sociais em perspectiva». História, Ciências, Saúde-Manguinhos, Rio de Janeiro, 21 (2), 385-402.

Armesto, X. A. (2005). «Notas teóricas en torno al concepto de postproductivismo agrario». Investigaciones Geográficas, 36, 137-156.

Arqué, M.; García, A. y Mateu, X. (1982). «La penetració del capitalisme a les comarques de l'Alt Pirineu». Documents d'Anàlisi Geogràfica, 1, 9-67.

Aurélie, A. (2007). La Via Campesina. La globalización y el poder del campesinado. Madrid: Popular.

Ayora, S. I. (2012). "Gastronomic Inventions and the Aesthetics of Regional Food». Etnofoor, Taste, 24 (2), 57-76.

Beck, U. (1998) World Risk Society. Cambridge: Polity Press.

Binimelis, R.; Bosch, M. y Herrero, A. (2008). A sol i serena. dones, món rural $i$ pagesia. Barcelona: Institut Català de les Dones. Generalitat de Catalunya.

Bové, J. y FrançOIs, D. (2003). La llavor del futur: l'agricultura explicada als ciutadans. Lérida: Pagès.

Bowler, I. R. (1996). "Agricultural land use and the post-productivist transition». En: López, A. y Molinero, F. (eds.). La investigación hispano-británica reciente en Geografía Rural: del campo tradicional a la transición postproductivista. Murcia: Asociación de Geógrafos Españoles, 179-187.

Brunet, I.; Pastor, I. y Belzunegui, A. (2002) Técnicas de investigación social: fundamentos epistemológicos y metodológicos. Barcelona: Pórtico.

Bryden, J. (2000). «Is there a New Rural Policy in OCDE countries?». Proceedings of the International Conference on Rural Communities and Identities in the Global Millennium. Nanaimo, Canada, 1-4 May 2000.

Burch, D. y Lawrence, G. (2009). "Towards a third Food Regime. Behind the transformation». Agriculture and Human Values, 26, 267-279. <http://dx.doi.org/10.1007/ s10460-009-9219-4>

Camarero, L. (1993). Del éxodo rural y del éxodo urbano. Ocaso y renacimiento de los asentamientos rurales en España. Madrid: Ministerio de Agricultura, Pesca y Alimentación.

Cloke, P. (2006). "Conceptualizing rurality». En: Cloke, P.; Marsden, T. y Mooney, P. H. (eds.). Handbook of Rural Studies. Londres: Sage, 18-28.

Contreras, J. y Ribas, J. (2012). «Los alimentos modificados. ¿El omnívoro desculturalizado?». Gazeta de Antropología, 28 (3), art. 04. 
Civitello, L. (2008). Cuisine and culture. A history of food and people. Hoboken, NJ: John Wiley \& Sons.

Díaz, C. (2005). ¿Cómo comemos? Cambios en los comportamientos alimentarios de los españoles. Madrid: Fundamentos.

Duch, G. (2010). Lo que hay que tragar: minienciclopedia de politica y alimentación. Madrid: Los Libros del Lince.

Euskadiko Gazteriaren Kontseilua (2010). "Jóvenes Baserritarras. Situación socioeconómica de la juventud agroganadera en la CAPV». Hizpideak, 4, 1-36.

FONTE, M. (2013). «Food consumption as social practice. Solidarity purchasing groups in Rome, Italy». Journal of Rural Studies, 32, 230-239.

GeIlfus, F. (2002). 80 herramientas para el desarrollo participativo: diagnóstico, planificación, monitoreo, evaluación. San José, Costa Rica: IICA.

Gillespie, J. R.; Gilbert, W. y Johnson, S. E. (2010). «Success in farm start-up in the Northeastern United States». Journal of Agriculture, Food Systems and Community Development, 1 (1), 31-48. <http://dx.doi.org/10.5304/jafscd.2010.011.008>

Green, J. y Thoroggod, N. (2009) [2004]. Qualitative methods for health research. Londres: Sage.

Guasch, O. (1996). Observación participante. Madrid: Centro de Investigaciones Sociológicas.

Guidonet, A. (2010). ¿Miedo a comer? Crisis alimentaria en contextos de abundancia. Barcelona: Icaria.

Guirado, C. y Tulla, A. F. (2010). «Entre l'abandonament i l'ús intensiu del territori? Sistema d'assentaments i gestió del territori en espais de muntanya. El cas de l'Alt Pirineu català». Documents d'Anàlisi Geogràfica, 56 (3), 607-623.

Hart, E. y Bond, M. (1995). Action research for health and social care: a guide to practice. Buckingham: Open University Press.

Holt, E. y Shattuck, A. (2011). "Food crises, food regimes and food movements: rumblings of reform or tides of transformation?». Journal of Peasant Studies, 38 (1), 109-144. <http://dx.doi.org/10.1080/03066150.2010.538578>

Ilbery, B. y Bowler, I. R. (1998). «From agricultural productivism to post-productivism». En: Ilbery, B. (ed.). The geography of rural change. Londres: Longman, 57-84.

Koch, T. y Kralik, D. (2006). Participatory action research in health care. Oxford: Blackwell.

Levenstein, H. (2012). Fear of Food: A History of why We Worry about what We Eat. Chicago: University of Chicago Press.

Lockwood, J. A. (1999). "Agriculture and biodiversity: finding our place in this world». Agriculture and Human Values, 16, 365-379.

López, D. y López, J. Á. (2003). Con la comida no se juega. Alternativas autogestionarias a la globalización capitalista desde la agroecología y el consumo. Madrid: Traficantes de Sueños.

López, I. (2014). Labelling the origin of food products. Towards sustainable territorial development? Wageningen: Ponsen \& Looijen.

López, I. y Aguilar, E. (2012). «Las etiquetas de calidad y el desarrollo territorial: los casos del queso de oveja merina de Grazalema y la carne de cordero de Texel». Revista de Economía Agrícola, 59 (2), 131-149.

- (2013). «La nueva economía rural europea. Especialización territorial de calidad en la Isla de Texel y la Sierra de Cádiz». Gazeta de Antropología, 29 (2), art. 04. 
López, I. y Pérez, A. (2014). «Las etiquetas de calidad agroalimentarias como herramientas de gobernablidad y desarrollo territorial: los casos del queso de oveja merina de Grazalema y la carne de cordero de Texel». Inguruak. Revista Vasca de Sociología y Ciencia Política. 57-58.

López, I. y Thомé, H. (2015). «Los pueblos mágicos como enclaves territoriales, el caso de Tequisquiapán y la Ruta del Queso y el Vino de Querétaro». En: Hernández, R. (coord.). Pueblos mágicos: discursos y realidades. Una mirada desde las políticas públicas y la gobernanza. México: Universidad Autónoma Metropolitana - Juan Pablos.

Lozano, C. y Aguilar, E. (2010). «Natural, tradicional y de la tierra. La promoción de la calidad agroalimentaria en los nuevos espacios rurales andaluces». En: Soler, M. y Guerrero, C. (eds.). PH Cuadernos. Patrimonio cultural en la nueva ruralidad andaluza. Sevilla: Junta de Andalucía y Consejería de Cultura, 127-139.

Mailfert, K. (2007). «New farmers networks: how beginning farmers build social connections in France». Tijdschrift voor Economische en Sociale Geografie, 98 (1), 21-31.

Marsden, T. (2000). «Food Matters and the Matter of Food: Towards a New Food Governance». Sociologia Ruralis, 40 (1), 20-29.

- (2003). The condition of rural sustainability. Wageningen: Royal Van Gorcum.

MedinA, F. X. (2010). "Introducció: reflexions sobre les alimentacions contemporànies». En: Reflexions sobre les alimentacions contemporànies. De les biotecnologies als productes ecològics. Barcelona: UOC, 11-22.

Medina, F. X. y Solanilla, L. (2013). «Patrimonio culinario, institucionalización y medios digitales». Ph Investigación, 1, 55-74.

$<$ www.iaph.es/phinvestigacion/index.php/phinvestigacion/article/view/13>

Menasche, R. (2003). «Frankenfoods e represetações sociais: percepções contemporâneas sobre biotecnologia, natureza e alimentação». Revista Theomai, 1 (4).

Milone, P.; Ventura, F. y Ye, J. (2015). Constructing a new framework for rural development. Bingley: Emerald Group Publishing Limited.

Monllor, N. (2011). Explorant la jove pagesia: camins, pràctiques i actituds en el marc d'un nou paradigma agrosocial. Estudi comparatiu entre el sud-oest de la provincia d'Ontario i les comarques gironines. Girona: Universitat de Girona. Tesis doctoral. <http://www.tdx.cat/handle/10803/70011>

- (2013). «El nuevo paradigma agrosocial, futuro del nuevo campesinado emergente». Polis, 34 (12), 203-223 <http://polis.revues.org/8831>

Monllor, N.; Guillamon, J.; Guirado, C.; Medina, F. X. y López, I. (2014). "Cocina, transformaciones sociales y nuevos conceptos para nuevas prácticas alimentarias: El caso de la Cuina compromesa (Burg, Pirineo de Lleida)». Studium, Humanidades y Ciencias Sociales, 20, 213-256.

Monllor, N.; Roca, A. y Ribas, A. (2005). Proposta i aplicació d'un model de contracte territorial al Pla de l'Estany. Gerona: Departament d'Agricultura, Ramaderia i Pesca - Càtedra de Pensament Territorial de la Universitat de Girona.

Morales, S. (2011). Som el que consumim? Escenaris i tendències en el consum de productes ecològics. Barcelona: UOC.

Murdoch, J.; Marsden, T. y Banks, J. (2000). "Quality, nature, and embeddedness: some theoretical considerations in the context of the food sector». Economic Geography, 76 (2), 107-125.

Nestle, M. (2007). Food politics. How the food industry influences nutrition and health. Los Ángeles: University of California Press. 
Niewolny, K. y Lillard, P. (2010). «Expanding the boundaries of beginning farmer training and program development: a review of contemporary iniciatives to cultivate a new generation of American farmers». Journal of Agriculture, Food Systems and Community Development, 1, 65-88.

Nützenadel, A. y Trentmann, F. (2008). Food and globalization. Consumption, markets and politics in the Modern World. Nueva York: Berg.

O'connor, D.; Renting, H.; Gorman, M. y Kinsella, J. (2006). «The Evolution of Rural Development in Europe and the Role of EU Policy». En: O'Connor, D.; Renting, H.; Gorman, M. y Kinsella, J. (eds.). Driving Rural Development Policy and Practice in Seven EU Countries. Assen: Royal van Gorcum, 1-30.

OCDE (2006). The New Rural Paradigm: policies and governance. París: OCDE.

Ogle, J. (2009). «Corn, Free Trade and the Mexican Quest for Food Security». Portland State University McNair Scholars Online Journal, 3, 154-183.

PADDock, J. (2015). «Invoking simplicity. Alternative food and the reinvention of distintion». Sociologia Ruralis, 55 (1), 22-40. <http://dx.doi.org/10.1111/soru.12056>

Pechlaner, G. y Otero, G. (2008). «The Third Food Regime Neoliberal Globalism and Agricultural Biotechnology in North America». Sociologia Ruralis, 48 (4), 351-371.

PÉrez, S. (2010). El retorno de los campesinos. Una oportunidad para nuestra supervivencia. Barcelona: Icaria.

Petrini, C. (2005). Buono, polito e giusto. Principi di nuova gastronomia. Turín: Einaudi.

Ploeg, J. D. van Der (2003). The virtual farmer: Past, present and future of the Dutch peasantry. Amsterdam: Van Gorcum.

- (2006). "Agricultural production in crisis». En: Cloke, P. J.; Marsden, T. y Mooney, P. (eds.). Handbook of Rural Studies. Londres: Sage, 258-277.

- (2008). The new peasantries: struggles for autonomy and sustainability in an era of empire and globalization. Londres: Routledge.

- (2010). "The food crisis, industrialized farming and the imperial regime». Journal of Agrarian Change, 10 (1), 98-106.

Ploeg, J. D. van Der, y Renting, H. (2004). «Behind the 'Redux', A Rejoinder to David Goodman». Sociologia Ruralis, 44 (2), 233-242.

Pollan, M. (2014). Cocinar. Una historia natural de la transformación. Barcelona: Debate.

Pujadas, J. J.; Comas d'Argemir, D. y Roca, J. (2010). Etnografía. Barcelona: UOC.

Regidor, J. (2008). Desarrollo rural sostenible: un nuevo desafío. Madrid: Ministerio de Medio Ambiente, Rural y Marino.

Renting, H.; Marsden, T. y Banks, J. (2003). "Understanding alternative food networks: exploring the role of short food supply chains in rural development». Environment and planning A, 35 (3), 393-412.

Rivera, G. y Tonatiuh, A. (2009). «El método de los estudios de caso». En: Reyes, G. (comp.). Acercamientos conceptuales y metodológicos para el estudio de la realidad agropecuaria y rural de México. Toluca: Universidad Autónoma del Estado de México, 257-282.

Romero, J. (2002). «Regulaciones agroambientales de la actividad agraria en la Unión Europea. Contexto, procesos y políticas públicas». En: Gómez, C. y González, J. (coord.). Agricultura y sociedad en el cambio de siglo. Madrid: McGraw Hill UNED, 623-652.

Rubio, P. (2010). «Modelización de los cambios y evolución reciente del sistema rural español». Boletín de la Asociación de Geógrafos Españoles, 54, 203-235. 
Scoтt, J. C. (1976). The Moral Economy of the Peasant: Subsistence and Rebellion in Southeast Asia. New Haven: Yale University Press.

Sgarbi, J. y Menasche, R. (2015). «Valorização de produtos alimentares tradicionais: os usos das indicações geográficas no contexto brasileiro». Cuadernos de Desarrollo Rural, 12 (75), 12-30.

Standage, T. (2009). An edible history of humanity. Nueva York: Walker \& Company.

VÁzquez, J. y Medina, F. X. (2015). «Migration, nostalgia and the building of a food imaginary: Mexican migrants at "La Pulga" Market in San Joaquin Valley, California». Journal for Communication Studies, 8 (2), 133-146.

Verbeke, W. (2005). "Agriculture and the food industry in the information age». European Review of Agricultural Economics, 32 (3), 347-368.

Viladomiu, L. (2003). «Noves tendències de desenvolupament rural a Europa». Quaderns Agraris, 28, 21-36.

Viladomiu, L. y Rosell, J. (2009). «Una política agraria europea (PAC) para el siglo XXI». Ecología Política, 38: 68-72.

Vivas, E. (2010). «El consum agroecològic a l'Estat espanyol: una opció política». En: Medina, F. X. (ed.). Reflexions sobre les alimentacions contemporànies. De les biotecnologies als productes ecològics. Barcelona: UOC, 149-174.

- (2014). El negocio de la comida ¿Quién controla nuestra alimentación? Barcelona: Icaria.

VV. AA. (2011). Corpus del patrimonio culinario catalán. Barcelona: RBA.

Woods, M. (2005). Rural Geography: processes, responses and experiences in rural restructuring. Londres: Sage. 